\title{
ESTUDO DA COMBUSTÃO IN-SITU EM RESERVATÓRIOS COM CARACTERÍSTICAS DO NORDESTE BRASILEIRO
}

\author{
M.L. ROCHA ${ }^{1}$,E. A. ARAÚJO ${ }^{1}$, J. L. M. BARILLAS ${ }^{1}$ \\ ${ }^{1}$ Universidade Federal do Rio Grande do Norte, Graduação em Engenharia de Petróleo \\ E-mail para contato: mirellinha_rocha@hotmail.com ; edsonandradesp@gmail.com; \\ jennys@eq.ufrn.br
}

\begin{abstract}
RESUMO - Com um número crescente de campos maduros, a recuperação de óleo pesado tem se apresentado um dos grandes desafios do setor petrolífero. O Nordeste Brasileiro, por exemplo, possui inúmeros reservatório de óleo pesado que possam vir a ser explorados com a utilização de métodos térmicos. Dentre os métodos de térmicos, a combustão in-situ (CIS) é uma das técnicas de recuperação de óleo na qual o calor e gerado dentro do reservatório. Dessa forma, este trabalho tem como objetivo realizar um estudo paramétrico do processo de combustão in-situ, usando um reservatório semi-sintético com características do Nordeste Brasileiro. Foi realizada uma análise de sensibilidade do processo para os parâmetros operacionais: concentração de oxigênio, vazão de injeção de ar e completação do poço injetor. Os resultados obtidos mostraram que a concentração de oxigênio foi o parâmetro que apresentou a maior influência a maior influência estatística no percentual de óleo recuperado.
\end{abstract}

\section{INTRODUÇÃO}

Em muitos reservatórios no mundo, o petróleo que se encontra nos reservatórios é pesado e possuem alta viscosidade o suficiente para tornar os métodos convencionais de recuperação ineficientes, ou seja, a injeção de água no reservatório não atinge nível de recuperação eficiente, ficando assim grande parte do óleo retido no reservatório.

Isso se deve ao fato da viscosidade do fluido injetado ser muito menor que a do fluido a ser deslocado, visto que o que possui menor viscosidade se move muito mais facilmente no meio poroso, encontrando caminhos preferenciais e se dirigindo rapidamente para outros poços de produtores, apresentando assim, uma eficiência de varrido baixa e sendo produzido rapidamente.

Dessa maneira o óleo fica retido, pois o fluido injetado não se desloca de forma apropriada dentro do reservatório, ficando grandes volumes de rocha nos quais o deslocamento não ocorreu. Assim, os métodos convencionais de recuperação tornam-se limitados, sendo necessárias novas alternativas de recuperação.Portanto, em reservatórios caracterizados pela presença de óleo pesado, os métodos de recuperação especiais tornam-se altamente aconselháveis do ponto de vista da eficiência de recuperação do óleo original.

A Combustão In-situ é uma técnica de recuperação térmica de óleo na qual o calor é produzido dentro do reservatório, contrastando com a injeção de fluidos previamente aquecidos, onde o calor é gerado na superfície e transportado para o reservatório por meio 
de um fluido. No processo in-situ, uma pequena porção do óleo do reservatório entra em ignição, a qual é sustentada pela injeção contínua de ar. Como em qualquer reação de combustão, o comburente (oxigênio) se combina com o combustível (óleo) liberando calor e formando produtos como água e dióxido de carbono para uma reação completa.

Nesse trabalho foi realizado um estudo da aplicação de combustão in-situ em um reservatório semi-sintético com características do Nordeste Brasileiro, com o objetivo de analisar a recuperação do óleo e a porcentagem de oxigênio consumido, quando submetido a diferentes parâmetros operacionais, tais como: vazões de injeção de ar, completação do poço injetor e concentração de oxigênio. Para o estudo, foi utilizado o simulador STARS ("Steam, Thermal, and Advanced Processes Reservoir Simulator"), do grupo CMG ("Computer Modelling Group”).

\section{METODOLOGIA}

No trabalho foi analisado um reservatório homogêneo com características do Nordeste Brasileiro. As dimensões da malha e as características do reservatório podem ser observadas na Tabela 1.

Tabela 1 - Modelo de Malha e propriedade da rocha-reservatório.

\begin{tabular}{|c|c|}
\hline Área do reservatório, $\left(\mathrm{m}^{2}\right)$ & $100 \times 100$ \\
\hline Espessura da zona de óleo, $\mathrm{h}(\mathrm{m})$ & 20 \\
\hline Profundidade do reservatório $(\mathrm{m})$ & 100 \\
\hline Contato água-óleo $(\mathrm{m})$ & 220 \\
\hline Número total de blocos & 4950 \\
\hline Número de blocos em $\mathrm{i}, \mathrm{j}, \mathrm{k}$ & $15,15,22$ \\
\hline Permeabilidade horizontal $(\mathrm{Kh}, \mathrm{mD})$ & 1000 \\
\hline Permeabilidade vertical $(\mathrm{Kv}, \mathrm{mD})$ & $0,1 \mathrm{x} \mathrm{Kh}$ \\
\hline Porosidade $(\%)$ & 30 \\
\hline Temperatura inicial $\left({ }^{\circ} \mathrm{F}\right)$ & 110 \\
\hline Pressão inicial $(\mathrm{psia})$ & $287(1978 \mathrm{kPa})$ \\
\hline
\end{tabular}

Na Figura 1, observa-se uma representação 3D do reservatório estudado. Pode-se perceber as dimensões do modelo e a localização do poço injetor vertical e do poço produtor. Neste sistema, a saturação inicial do óleo no topo do reservatório é 0,70 . 


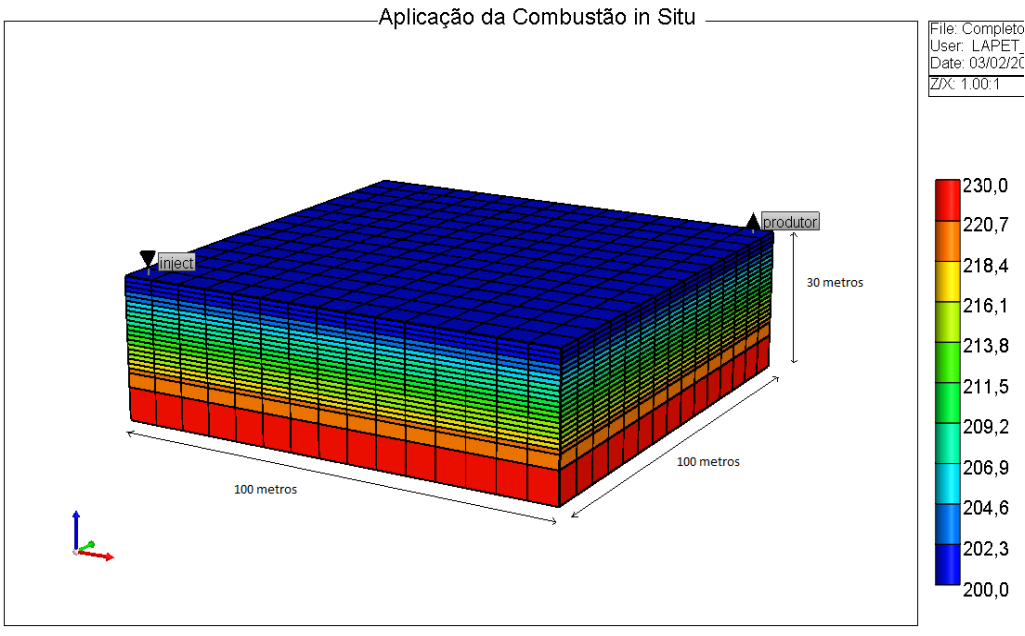

Figura 1 - Vista 3D do reservatório.

Na Figura 2 a seguir mostra as diferentes formas de completação do poço injetor.

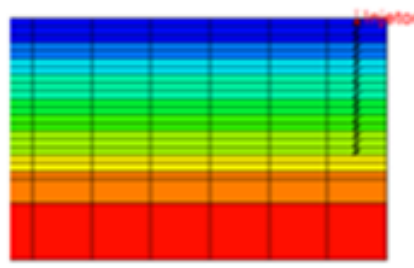

Completação Topo

Topo $(1,15,1)$

Base $(1,15,17)$

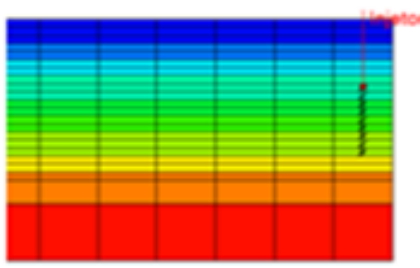

Completação Meio

Topo $(1,15,9)$

Base $(1,15,17)$

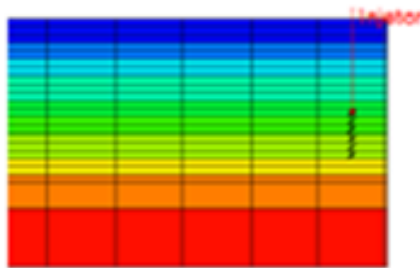

Completação Inferior

Topo $(1,15,12)$

Base $(1,15,17)$

Figura 2 - Configuração da completação do poço injetor.

$\mathrm{O}$ modelo de fluido utilizado apresenta 5 pseudo-componentes e 2 componentes: óleo pesado $\left(\mathrm{C}_{21} \mathrm{C}_{40+}\right)$, óleo médio $\left(\mathrm{C}_{13} \mathrm{C}_{20}\right)$, óleo leve $\left(\mathrm{C}_{6}-\mathrm{C}_{12}\right)$, gás inerte $\left(\mathrm{CO}_{2}\right.$ e $\left.\mathrm{N}_{2}\right)$, coque $(\mathrm{C})$, oxigênio $\left(\mathrm{O}_{2}\right)$ e água $\left(\mathrm{H}_{2} \mathrm{O}\right)$, respectivamente. A Tabela 2 apresenta as composições.

Tabela 2 - Agrupamentos e a fração mássica inicial.

\begin{tabular}{|c|c|}
\hline Componentes & Fração Mássica Inicial \\
\hline $\mathrm{H}_{2} \mathrm{O}$ & 0 \\
\hline $\mathrm{CO}_{2}-\mathrm{N}_{2}$ & 0 \\
\hline $\mathrm{O}_{2}$ & 0 \\
\hline Óleo leve $\left(\mathrm{C}_{6}-\mathrm{C}_{12}\right)$ & 0,10 \\
\hline Óleo médio $\left(\mathrm{C}_{13}-\mathrm{C}_{20}\right)$ & 0,20 \\
\hline Óleo pesado $\left(\mathrm{C}_{21}-\mathrm{C}_{40+}\right)$ & 0,70 \\
\hline Coque $(\mathrm{C})$ & 0 \\
\hline
\end{tabular}


As reações químicas consideradas no sistema foram: 1-3: Oxidação,4-5: Craqueamento do óleo pesado $\left(\mathrm{C}_{21}-\mathrm{C}_{40+}\right)$ e óleo médio $\left(\mathrm{C}_{13}-\mathrm{C}_{20}\right)$, 6: oxidação do coque (C).

- Reação 1: $\mathrm{C}_{21}-\mathrm{C}_{40+}+52 \mathrm{O}_{2} \rightarrow 36 \mathrm{CO}_{2}+32 \mathrm{H}_{2} \mathrm{O}$

- Reação 2: $\mathrm{C}_{13}-\mathrm{C}_{20}+25 \mathrm{O}_{2} \rightarrow 16,5 \mathrm{CO}_{2}+17,5 \mathrm{H}_{2} \mathrm{O}$

- Reação 3: $\mathrm{C}_{6}-\mathrm{C}_{12}+14 \mathrm{O} 2 \rightarrow 9 \mathrm{CO}_{2}+10 \mathrm{H}_{2} \mathrm{O}$

- Reação 4: $\mathrm{C}_{21}-\mathrm{C}_{40+} \rightarrow \mathrm{C}_{13}-\mathrm{C}_{20}+1,5 \mathrm{C}_{6}-\mathrm{C}_{12}+6$ Coque $+\mathrm{CO}_{2}$

- Reação 5: $\mathrm{C}_{13}-\mathrm{C}_{20} \rightarrow \quad 1,75 \mathrm{C}_{6}-\mathrm{C}_{12}+1,2 \mathrm{Coque}+\mathrm{CO}_{2}$

- Reação 6: Coque $+1,25 \mathrm{O} 2 \rightarrow \mathrm{CO}_{2}+0,5 \mathrm{H}_{2} \mathrm{O}$

Os coeficientes estequiométricos das reações químicas foram ajustados no módulo "Builder" da CMG. A energia de ativação e a entalpia das reações químicas usadas neste trabalho foram adaptadas das publicadas pelos autores Rojas et al. 2010, isto devido à falta de informação na literatura destes parâmetros, para os diferentes componentes de óleo, e podem ser observadas na Tabela 3.

Tabela 3 - Dados do modelo de reação.

\begin{tabular}{|l|c|c|}
\hline & Energia de Ativação $(\mathrm{kJ} / \mathrm{kg})$ & Entalpia da Reação $(\mathrm{kJ} / \mathrm{kg})$ \\
\hline Reação 1 & 76258 & $1,89 \times 10^{6}$ \\
\hline Reação 2 & 76258 & $1,05 \times 10^{7}$ \\
\hline Reação 3 & 76258 & $4,88 \times 10^{6}$ \\
\hline Reação 4 & 66989 & $4,65 \times 10^{4}$ \\
\hline Reação 5 & 66989 & $4,65 \times 10^{4}$ \\
\hline Reação 6 & 66989 & $5,34 \times 10^{5}$ \\
\hline
\end{tabular}

A Tabela 4 apresenta como as variáveis operacionais foram analisadas.

Tabela 4 - Parâmetros operacionais trabalhados para as diferentes completações.

\begin{tabular}{|c|c|c|}
\hline Completação Topo & Completação Meio & Completação Inferior \\
\hline $\mathrm{Q}=15.000$ e $21 \% \mathrm{O} 2$ & $\mathrm{Q}=15.000$ e $21 \% \mathrm{O} 2$ & $\mathrm{Q}=15.000$ e $21 \% \mathrm{O} 2$ \\
\hline $\mathrm{Q}=20.000$ e $21 \% \mathrm{O} 2$ & $\mathrm{Q}=20.000$ e $21 \%$ O2 & $\mathrm{Q}=20.000$ e $21 \%$ O2 \\
\hline $\mathrm{Q}=25.000$ e $21 \% \mathrm{O} 2$ & $\mathrm{Q}=25.000$ e $21 \%$ O2 & $\mathrm{Q}=25.000$ e $21 \%$ O2 \\
\hline $\mathrm{Q}=15.000$ e $50 \%$ O2 & $\mathrm{Q}=15.000$ e $50 \%$ O2 & $\mathrm{Q}=15.000$ e $50 \%$ O2 \\
\hline $\mathrm{Q}=20.000$ e $50 \%$ O2 & $\mathrm{Q}=20.000$ e $50 \%$ O2 & $\mathrm{Q}=20.000$ e $50 \%$ O2 \\
\hline $\mathrm{Q}=25.000$ e $50 \%$ O2 & $\mathrm{Q}=25.000$ e $50 \%$ O2 & $\mathrm{Q}=25.000$ e $50 \%$ O2 \\
\hline
\end{tabular}

A partir dos resultados simulados obtidos para tais parâmetros operacionais, foram feitas análises do fator de recuperação e também a porcentagem de oxigênio consumido para alguns casos. 


\section{RESULTADOS E DISCUSSÕES}

A Tabela 5 mostra o intervalo dos parâmetros estudados. Os níveis são representados por valores mínimos $(-1)$, valores intermediários $(0)$ e valores máximos $(+1)$. No decorrer da análise dos parâmetros operacionais as características que não interessavam para estudo foram fixadas.

Tabela 5 - Níveis estudados

\begin{tabular}{|c|ccc|}
\hline \multicolumn{4}{|c|}{ Níveis } \\
\hline Parâmetros & -1 & 0 & 1 \\
Vazão de Injeção (m³/dia) & 15.000 & 20.000 & 25.000 \\
Completação do poço Injetor & Topo & Meio & Inferior \\
Concentração de Oxigênio (\%) & $21 \%$ & - & $50 \%$ \\
\hline
\end{tabular}

Na Tabela 6, contém os 18 casos simulados para o modelo de fluido denominado Nordeste Brasileiro com o percentual de óleo recuperado em 20 anos de produção. Os casos simulados estão distribuídos de forma decrescente de \%FR em 20 anos de produção.

Tabela 6 - Fator de recuperação para casos simulados

\begin{tabular}{|c|c|c|c|c|c|c|c|}
\hline & C.Oxigênio & Vazão & Completação & $\begin{array}{c}\text { FR }(\%) \\
\text { 5anos }\end{array}$ & $\begin{array}{c}\text { FR (\%) } \\
\text { 10anos }\end{array}$ & $\begin{array}{c}\text { FR (\%) } \\
\text { 15anos }\end{array}$ & $\begin{array}{l}\text { FR }(\%) \\
20 \text { anos }\end{array}$ \\
\hline Caso 13 & $21 \%$ & 15000 & Inferior & 2,38 & 17,00 & 58,12 & 63,09 \\
\hline Caso 1 & $21 \%$ & 15000 & Topo & 2,09 & 5,68 & 59,00 & 63,00 \\
\hline Caso 7 & $21 \%$ & 15000 & Meio & 2,22 & 10,27 & 58,00 & 62,89 \\
\hline Caso 14 & $21 \%$ & 20000 & Inferior & 2,38 & 43,46 & 59,00 & 59,77 \\
\hline Caso 2 & $21 \%$ & 20000 & Topo & 2,05 & 43,10 & 57,81 & 59,12 \\
\hline Caso 8 & $21 \%$ & 20000 & Meio & 2,22 & 42,92 & 58,00 & 59,00 \\
\hline Caso 15 & $21 \%$ & 25000 & Inferior & 6,27 & 52,59 & 56,77 & 57,16 \\
\hline Caso 9 & $21 \%$ & 25000 & Meio & 5,55 & 52,24 & 55,73 & 56,37 \\
\hline Caso 3 & $21 \%$ & 25000 & Topo & 4,50 & 52,64 & 55,00 & 56,01 \\
\hline Caso 16 & $50 \%$ & 15000 & Inferior & 20,06 & 52,86 & 53,73 & 54,44 \\
\hline Caso 10 & $50 \%$ & 15000 & Meio & 19,60 & 51,73 & 52,62 & 53,83 \\
\hline Caso 4 & $50 \%$ & 15000 & Topo & 19,06 & 49,87 & 51,14 & 52,90 \\
\hline Caso 17 & $50 \%$ & 20000 & Inferior & 33,35 & 50,47 & 51,87 & 52,17 \\
\hline Caso 18 & $50 \%$ & 25000 & Inferior & 41,12 & 49,28 & 51,07 & 51,31 \\
\hline Caso 11 & $50 \%$ & 20000 & Meio & 33,12 & 48,64 & 49,95 & 50,84 \\
\hline Caso 5 & $50 \%$ & 20000 & Topo & 32,82 & 46,65 & 48,17 & 49,56 \\
\hline Caso 12 & $50 \%$ & 25000 & Meio & 40,58 & 47,32 & 48,98 & 49,48 \\
\hline Caso 6 & $50 \%$ & 25000 & Topo & 39,43 & 45,49 & 46,94 & 47,97 \\
\hline
\end{tabular}

Para análise dos parâmetros operacionais foi utilizado o diagrama de Pareto, para verificar o efeito de cada variável e das interações, na recuperação de óleo em 20 anos, isto pode ser observado na Figura 3. 


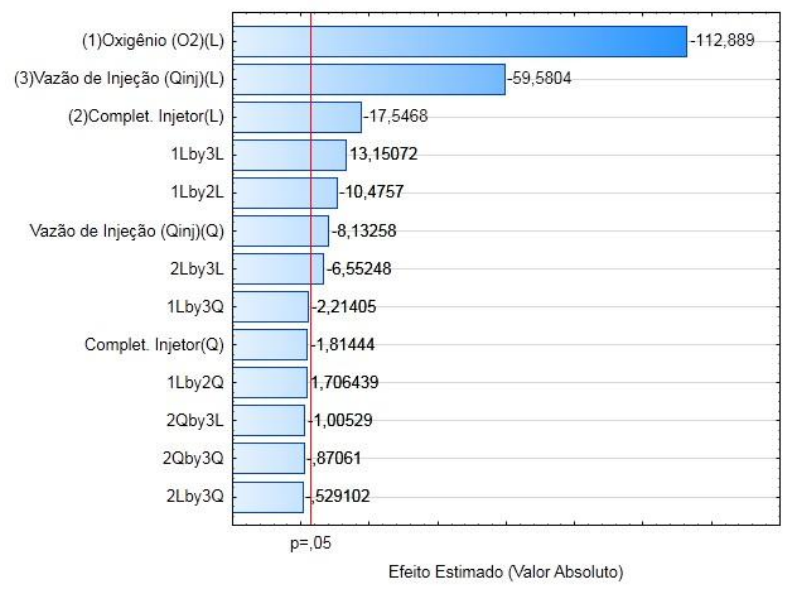

Figura 3 - Diagrama de Pareto das variáveis operacionais- Função Objetivo: óleo recuperado em 20 anos.

Na Figura 3, observa-se em ordem decrescente a importância dos efeitos individuais e das interações: concentração de oxigênio $\left(\% \mathrm{O}_{2}\right)$, vazão de injeção de ar (Qinj) e Completação Injetor, sendo os três primeiros de maior importância. Pode ser observado que a mudança do nível na concentração de oxigênio $\left(\% \mathrm{O}_{2}\right)$ do mínimo para o máximo há um incremento na recuperação de óleo em vinte anos, o mesmo acontece quando é analisada a vazão de injeção de ar (Qinj), a análise da interação entre estes parâmetros é estudada através da Figura 4. O parâmetro completação dos poços mostra um efeito de menor sensibilidade na recuperação de óleo em vinte anos, mas ainda significativo.

Na Figura 4 pode ser observada a superfície de resposta das variáveis: concentração de oxigênio $\left(\% \mathrm{O}_{2}\right)$ e vazão de injeção de ar (Qinj) e a função objetivo estudada foi \%FR em 20 anos.

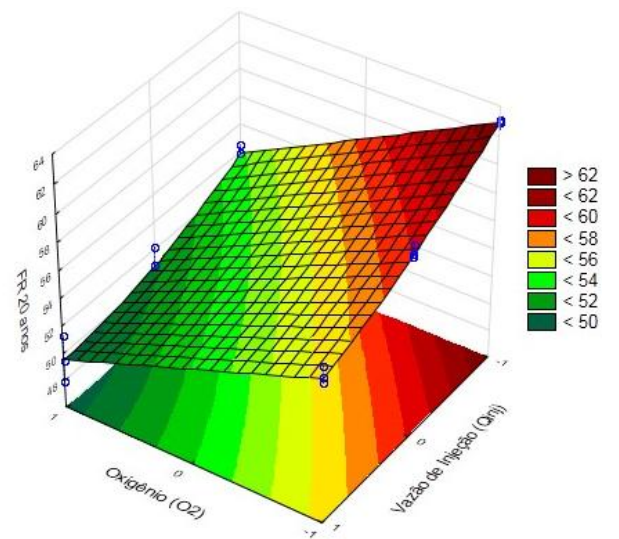

Figura 4 - Superfície de resposta da vazão de injeção de ar e a concentração de oxigênio para 20 anos.

Nesta Figura 4, observa-se que quando os parâmetros vazão de injeção de ar e concentração de oxigênio estão no nível mínimo (Qinj: $15.000 \mathrm{~m}^{3} \mathrm{std} / \mathrm{dia}$ e \%O2: 21) a recuperação de óleo é maior. Já quando há um incremento da vazão de ar e da concentração de oxigênio acontece uma queda na recuperação de óleo em 20 anos, essa queda é possivelmente devido à maior quantidade de gás no sistema o que passa a ser produzido junto com o óleo, comprometendo assim a produção do mesmo. 
Foi realizada análise da porcentagem de oxigênio consumida pelo sistema em 20 anos para $21 \%$ e $50 \%$ com uma vazão de ar 25.000 m³/dia e completação do poço injetor (inferior). Esta análise foi auxiliada pela Figura 5 que representa o gráfico da produção acumulada de oxigênio versus tempo.

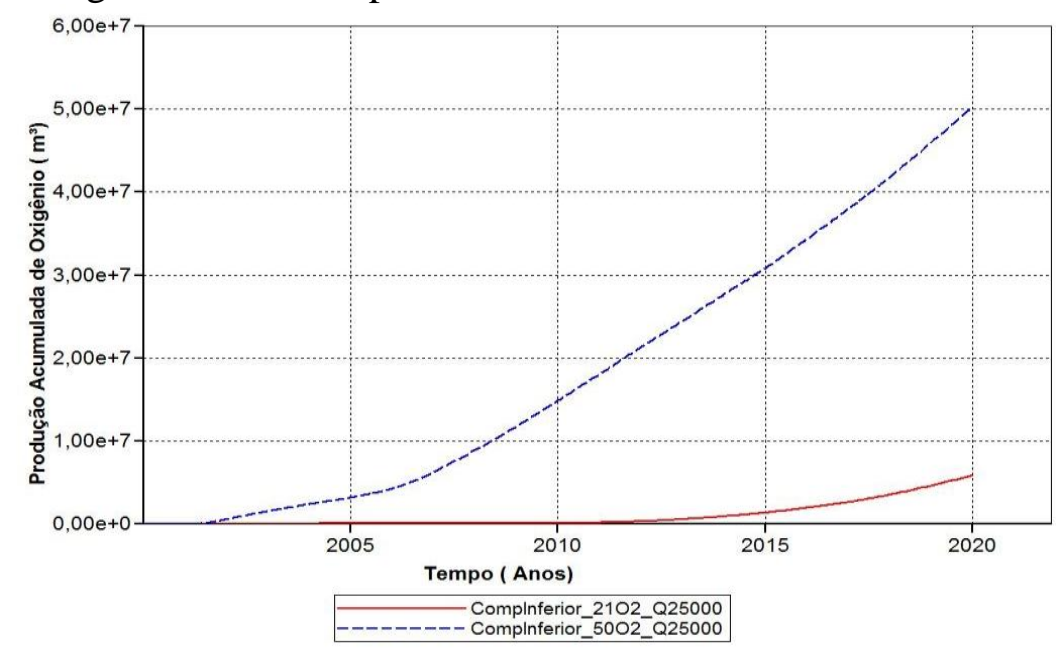

Figura 5 - Produção acumulada de gás oxigênio para completação inferior com diferentes concentrações de oxigênio e uma vazão de injeção de ar 25.000 m³/dia.

Para analisar o consumo de oxigênio foi verificada a produção acumulada de gás em função do tempo para o componente oxigênio (injetado e produzido). A Figura 5 mostra a quantidade de oxigênio produzido no decorrer dos 20 anos de projeto. A Eq.1 11 representa a porcentagem de oxigênio consumido.

$\% \mathrm{O}_{2}$ Consumido $=\frac{\mid O_{2} \text { Produzido- } O_{2} \text { Injetado } \mid}{O_{2} \text { Injetado }}$

Onde:

- $\mathrm{O}_{2}$ Produzido: Volume de Gás Oxigênio Produzido

- $\mathrm{O}_{2}$ Injetado: Volume de Gás Oxigênio Injetado

- $\% \mathrm{O}_{2}$ Consumido: Porcentagem de Oxigênio Consumido

Na Eq.1 1 a quantidade de oxigênio produzido que é obtido a partir da Figura 5, e a porcentagem de oxigênio injetado que depende da vazão de injeção de ar e da concentração de oxigênio.

Juntamente com a Figura 5 e Eq.1 1, foi possível obter a porcentagem de oxigênio consumido, como mostrado na Tabela .

Tabela 7 - Valores de oxigênio injetado, produzido e porcentagem de oxigênio consumido.

\begin{tabular}{|c|c|c|}
\hline Resultados 20anos de produção & $\begin{array}{c}\mathbf{5 0 \%} \mathbf{~ d e ~} \mathbf{~ O 2} \\
\mathbf{Q = 2 5 . 0 0 0} \mathbf{~ m}^{\mathbf{3}} / \mathbf{d i a}\end{array}$ & $\begin{array}{c}\mathbf{2 1 \%} \mathbf{~ d e ~ O 2} \\
\mathbf{Q = 2 5 . 0 0 0} \mathbf{~ m}^{\mathbf{3}} / \mathbf{d i a}\end{array}$ \\
\hline O2 Injetado $\left(\mathbf{m}^{\mathbf{3}}\right)$ & 91.250 .000 & 38.325 .000 \\
\hline O2 Produzido $\left(\mathbf{~ m}^{\mathbf{3}}\right)$ & 50.968 .162 & 5.921 .103 \\
\hline O2 Consumido $(\boldsymbol{\%})$ & 44,14 & 84,55 \\
\hline FR $(\boldsymbol{\%})$ & 51,31 & 57,16 \\
\hline
\end{tabular}


Observa-se que há uma variação da porcentagem de oxigênio consumido entre as diferentes concentrações de oxigênio, o que pode ser observado na Tabela 7Error! Reference source not found.. O caso com uma maior concentração de oxigênio (50\%) no ar mostrou um menor consumo do mesmo em relação à concentração de oxigênio $21 \%$. Esse parâmetro foi importante para observar que injetando ar enriquecido foram consumidos $44,14 \%$ pela frente de calor e restante produzido. Para a concentração de 21 $\%$ de oxigênio são consumidos na reação química em vinte anos $84,55 \%$, portanto o seu consumo é bem maior quando comparado com a concentração de $50 \%$ de oxigênio, logo a saturação de gás é menor comprometendo menos a produção de óleo.

\section{CONCLUSÕES}

As principais conclusões deste estudo são:

- A concentração de oxigênio foi o parâmetro que apresentou a maior influência no óleo recuperado em 20 anos.

- Dentre as configurações da completação do poço injetor, a que possibilitou uma maior recuperação de óleo em diferentes anos, foi a completação inferior.

- A concentração de oxigênio e a vazão de injeção de ar em nível mínimo ( $21 \%$ e $15.000 \mathrm{~m}^{3} \mathrm{std} /$ dia) obtiveram maior recuperação de óleo em 20 anos.

- A porcentagem de oxigênio consumido é maior para o modelo de menor concentração de oxigênio (21\%).

\section{AGRADECIMENTOS}

À CMG (Computer Modelling Group) pelo programa, ao PRH-PB 221 e a Petrobrás pelo apoio financeiro.

\section{REFERÊNCIAS}

ARAÚJO, E.A. - Estudo do processo de combustão in-situ usando poços horizontais como produtores de óleo (toe-to-heel air injection). Dissertação de Mestrado, UFRN, Programa de Pós-graduação em Ciência e Engenharia de Petróleo, Natal-RN, 2012a.

ARAÚJO, J. S. Estudo paramétrico do processo de combustão in situ como método de recuperação avançada de petróleo. Tese de Doutorado, UFRN ,Programa de PósGraduação em Ciência e Engenharia de Petróleo. Natal-RN,Brasil, 2012b.

BARILLAS, J.L.M.; Estudo do Processo de Drenagem Gravitacional de Óleo com Injeção Contínua de Vapor em Poços Horizontais. Dissertação de Mestrado em Engenharia Química, Universidade Federal do Rio Grande do Norte, 2005. 